\title{
Telemedicine-assisted treatment of patients with intracerebral hemorrhage
}

\author{
Filippo F. Angileri, M.D., Ph.D., Salvatore Cardali, M.D., Ph.D., \\ Alfredo Conti, M.D., Ph.D., Giovanni Raffa, M.D., and Francesco Tomasello, M.D.
}

Department of Neurosurgery, University of Messina, Italy

\begin{abstract}
Object. Telemedicine provides a new approach to improve stroke care in community settings, delivering acute stroke expertise to hospitals in rural areas. Given the controversies in many aspects of the treatment of intracerebral hemorrhage (ICH) and the lack of guidelines, a prompt neurosurgical second opinion may facilitate the treatment of patients with ICH. Here, the authors' 8-year experience with the use of telemedicine in the management of ICH is reported.

Methods. The medical records of patients with $\mathrm{ICH}$ treated through a telemedicine system in the district of Messina, Italy, between June 2003 and June 2011 were retrospectively reviewed. Neuroradiological and clinical data for patients were transmitted through a high-technology "hub-and-spoke" telemedicine network. Neurosurgical teleconsulting (at the hub) was available for 7 peripheral hospitals (spokes) serving about 700,000 people. The authors analyzed 1) the time between peripheral hospital admission and the specialized second opinion consultation, 2) primary and secondary transfers to the authors' neurosurgery department, and 3 ) the treatments (surgical or medical) of patients transferred to the hub.

Results. The telemedicine network was used to treat more than 2800 patients, 733 with ICH. A neurosurgical consultation was provided in 38 minutes versus 160 minutes for a consultation without telemedicine. One hundred seventy-six (24\%) of 733 patients were primarily transferred to the hub. Ninety-five patients (13\%) underwent surgical treatment. The remaining 81 patients $(11 \%)$ underwent neurointensive care. Eight (1.4\%) of 557 patients treated at the spokes needed a secondary transfer for surgical treatment because of a worsening clinical condition and/or CT findings. Considering secondary and inappropriate transfers, the interpretation of data was correct in $96.5 \%$ of cases.

Conclusions. Telemedicine allowed rapid visualization of neuroradiological and clinical data, providing neurosurgical expertise to community hospitals on demand and within minutes. It allowed the treatment of patients at peripheral hospitals and optimized resources. A small percentage of patients treated at the peripheral hospitals had secondary deterioration. Telemedicine allowed fast patient transfer when necessary and provided improved accuracy in patient care.

(http://thejns.org/doi/abs/10.3171/2012.1.FOCUS11356)
\end{abstract}

KEY WordS • intracerebral hemorrhage • telemedicine • telestroke

I

NTRACEREBRAL hemorrhage accounts for $15 \%-30 \%$ of all acute strokes, with an incidence of approximately $12-15$ cases/100,000 persons/year..$^{5,10}$ Overall mortality from ICH is worse than that from ischemic stroke, approaching $50 \%$ at 30 days after hemorrhage, ${ }^{6,9}$ and patients who survive are independent at 6 months after ICH in less than $20 \%$ of cases. ${ }^{5}$ Because of its frequency and dramatic impact on the neurological status of patients, $\mathrm{ICH}$ is one of the most frequent clinical conditions for

\footnotetext{
Abbreviations used in this paper: CTA = CT angiography; GCS = Glasgow Coma Scale; ICH = intracerebral hemorrhage; ICP = intracranial pressure; IVH = intraventricular hemorrhage; MRA = MR angiography; PACS = picture archiving and communication system; ROI = region of interest.
}

which an emergency neurosurgical consultation is required. An expert opinion is needed to coordinate the best patient care, because there are no guidelines for the management of many aspects of $\mathrm{ICH}$, including the choice of medical versus surgical treatment. Telemedicine is the ideal means of immediately providing this consultation for patients admitted to peripheral hospitals. ${ }^{12}$

"Telemedicine," a term coined in the $1970 \mathrm{~s},{ }^{25}$ is the use of information and communication technology to improve patient outcomes by increasing access to care, medical information, and evidence-based clinical practice. It includes consultative, diagnostic, and treatment services with the aim of improving patient care and the efficacy of the diagnostic and therapeutic strategies at the peripheral hospitals. 
Many studies have shown that telemedicine improves the care of patients with ischemic stroke who have been admitted to peripheral hospitals, ${ }^{12-14,22,27}$ allowing for the quick selection of candidates for thrombolytic ther$a^{7} y^{7}$ and eventually improving the long-term neurological outcome. ${ }^{3,18}$ However, studies focused on the role of telemedicine in the treatment of patients with $\mathrm{ICH}$ in an acute setting are missing. The aim of the present study was to analyze the role of a telemedicine network in the treatment of patients with spontaneous ICH in terms of a faster neurosurgical evaluation and selection of patients who are the best candidates for direct neurosurgical or neurointensive care.

\section{Methods}

A telemedicine network project named RESPECT, from the Italian "REte SPECialistica per il Trauma," meaning specialized network for trauma, was set up in 2003 and co-financed by the Italian Ministry of Education, University and Research. The telemedicine system is coordinated by the Department of Neurosurgery at the University of Messina. Seven peripheral hospitals are connected to this neurosurgical department. The system serves a population of 684,703 people living in the area of Messina, whose extra-urban territory is mainly composed of a rural territory and 7 small islands. We retrospectively reviewed all medical records of patients with spontaneous ICH who had been admitted to the peripheral hospitals of the Messina district between June 2003 and June 2011 and for whom an urgent neurosurgical consultation had been requested.

\section{Teleconsultation System and Procedures}

Neuroradiological data on patients were transmitted from the peripheral hospitals to our department through a high-technology "hub-and-spoke" telemedicine network implemented in the territory of the Messina province. The radiology departments of the connected hospitals (the "spokes") used PACS (picture archiving and communication system) technology for archiving the images. The images were reviewed at the Department of Neurosurgery at the University of Messina (the "hub").

The transmission of information was performed using a pixels-on-demand technology provided by Telbios S.p.A. In brief, the system encodes only the pixels relevant for presenting the required image view, namely a $\mathrm{ROI}$ requested by the user and not the full image file, by connecting to the original archived images. The ROI is streamed to the client location from the original PACS in lossless quality and in real time. Once the hub user selects a remote image from the archive, the system performs a fast preprocessing step in near real time. From then on, the system is able to respond to any request in real time. When a ROI request arrives at the server, progressive image encoding is performed only for the ROI and not for the full image. Similarly, on the client side, decoding and rendering is only performed for the ROI. When a user pans, zooms, or scrolls to the next slices, the server encodes the missing parts of the new ROI "on the fly" and sends them to the client.

The system is connected to the DICOM (Digital Im- aging and Communications in Medicine) archive via a fast LAN (local area network) connection, and a further integrated services delivery network line is provided in case of malfunctioning. Images are taken via DICOM protocol or from direct file system access (read-only input/output).

When a patient with $\mathrm{ICH}$ was admitted to the spoke hospital, he or she underwent $\mathrm{CT}$ scanning. The radiologist on duty interpreted the CT images. A neurosurgical consultation was requested through the telemedicine system. The neurosurgeon on duty at the academic hub analyzed the neuroradiological images along with the radiology report, as well as anamnestic and clinical data. Clinical information included age, sex, GCS score, pupillary status, respiratory status, complete blood count, coagulation status, and use of anticoagulants, heparin, or antiplatelet drugs. Any information concerning clinical onset and evolution of symptoms or previous relevant pathological events were communicated and recorded as well.

After neurosurgical consultation, patients were treated at the peripheral hospitals or transferred to the hub for the best treatment (medical or surgical), depending on the case. Patients were treated at the peripheral hospital through clinical observation, neuroradiological monitoring, and medical therapy or were transferred to our department for clinical/neuroradiological monitoring and medical/surgical treatment.

We analyzed the 1) time between peripheral hospital admission and the second opinion specialist consultation, 2) primary and secondary transfers to our neurosurgical department, and 3) treatment (surgical or medical) of patients transferred to the hub. Through the analysis of the aforementioned parameters, we sought to investigate the utility of our telemedicine network in terms of faster transfer to a specialized hospital, better management at the peripheral hospitals, and improved medical and/or surgical treatment of patients with hemorrhagic stroke.

\section{Results}

Seven hundred thirty-three patients with $\mathrm{ICH}$ received neurosurgical consultation through the Telbios telemedicine system at the neurosurgical clinic of the University of Messina between June 2003 and June 2011. These patients with ICH represented $26 \%$ of all patients who needed an urgent neurosurgical consultation through telemedicine during the selected period (2819 patients).

The first end point of this study was the analysis of the time frame needed to provide a neurosurgical second opinion consultation. Analyzing the CT scanning records and the relevant neurosurgical opinion, we recorded a mean of 38 minutes (range 23-109 minutes) between hospital admission at the spoke and neurosurgical consultation at the hub. The mean time was 160 minutes (range 68-204 minutes) for a second opinion consultation obtained through patient transportation, before the introduction of the telemedicine system. The time needed to obtain neurosurgical teleconsultation at the periphery was not dissimilar to that in Messina's urban area, which was estimated at an average of 26 minutes (range 0-37 minutes). 
The second study end point was the analysis of primary and secondary transfers to our neurosurgical department and neurointensive care. Data are summarized in Fig. 1. During the evaluation period, 176 (24\%) of 733 patients needed a primary transfer to the academic hub for the management of ICH. Selecting patients for transfer was based on a combination of clinical, demographic, and radiological characteristics. Hematoma location, CT features, and size were the radiological characteristics considered in combination with the age of, relevant clinical data on, and neurological status of the patient. In cases of controversial interpretation, the neuroradiological team at the hub reevaluated the images.

On the basis of hematoma location, capsular, thalamic, or posterior fossa ICH in patients older than 45 years and with a history of systemic hypertension was presumptively judged as primary hypertensive $\mathrm{ICH}$, without the need for further diagnostic workup. Transfer to the hub of patients with ICHs in the aforementioned locations was done on the basis of hematoma size and patient clinical status (Fig. 1). Patients with capsular or thalamic ICHs with volumes ranging between 30 and 70

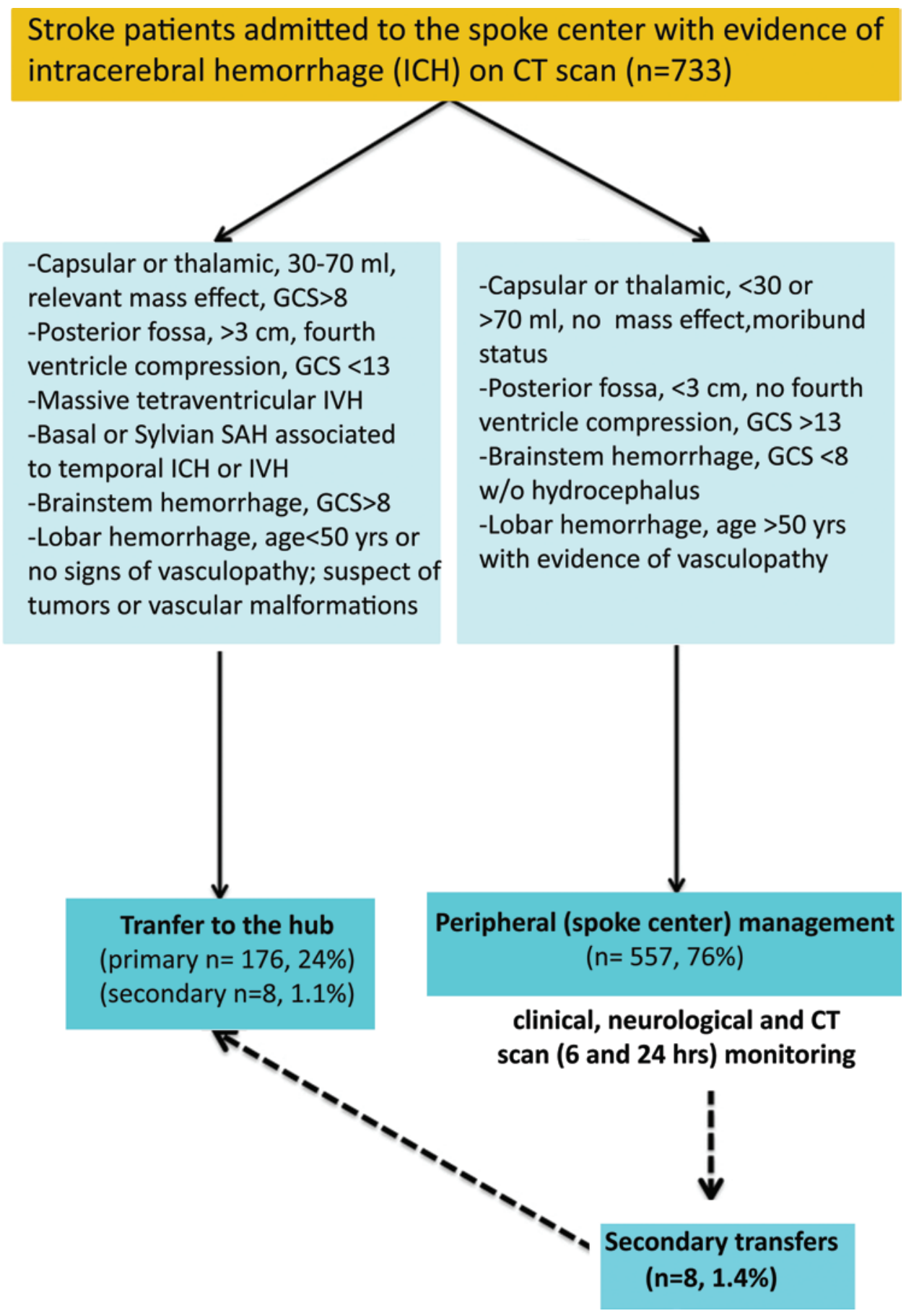

FIG. 1. Algorithm summarizing referral strategies for patient selection. $\mathrm{SAH}=$ subarachnoid hemorrhage. 
$\mathrm{ml}$ - that is, with evidence of relevant mass effect-and a GCS score $>8$ were transferred to the hub and underwent surgical treatment (Fig. 2). With regard to posterior fossa ICHs, compression of the fourth ventricle and/or a clot size $>3 \mathrm{~cm}$ were considered criteria for transfer to our neurosurgical unit. Patients underwent surgical treatment if they had a GCS score $<13$ or evidence of neurological deterioration.

Patients with blood in the entire ventricular system were considered at risk for hydrocephalus and were centralized, whereas patients with only a minimal amount of blood in 1 lateral ventricle were left at the peripheral hospital and observed. All patients with a subarachnoid clot in the basal or sylvian cisterns associated with temporal or IVH were admitted to the central hospital for further studies. Patients with brainstem hemorrhage were admitted only if the GCS score was $>8$ to treat or prevent deterioration as a result of acute hydrocephalus. Patients in a deep coma because of large brainstem injuries were treated only with supportive care.

With regard to lobar hemorrhages, patients younger than 50 years of age were admitted to the central hospital (Fig. 3). Older patients with evidence of vasculopathy remained at the peripheral hospital with the indication for performing MRI and MRA.

In general, other CT features were considered, including marks suggestive of vascular abnormalities, tumors, sinus thrombosis, or hemorrhagic transformation of ischemic stroke, and were evaluated on an individual basis. When a secondary ICH was suspected, the patient was ad- mitted to the neurosurgical department to complete our institutional diagnostic workup protocol consisting of CTA, MRI, MRA, SPECT, and eventually angiography. ${ }^{20}$

The 557 patients who remained at a peripheral hospital had neuroradiological images and clinical/neurological conditions that did not require direct neurosurgical or neurointensive care management. They were treated through clinical, neurological, and neuroradiological monitoring, according to the neurosurgical consultation and indications. In particular, the neurosurgeon recommended a control CT scan 6 hours later and a second control scan 24 hours after stroke onset. This practice was different for patients directly admitted to the central hospital, who did not undergo the 6-hour CT control study unless their condition clinically deteriorated.

Eight (1.4\%) of 557 patients needed a secondary transfer to our department for a worsening of clinical conditions and/or deterioration of neuroradiological findings. Five of these 8 patients were on anticoagulation drugs for other diseases. Patients in a deep coma or with a moribund status were not transferred, however, and were given only supportive care.

Of the 176 patients who were centralized, 95 (13\% of the overall group) underwent surgical evacuation of an ICH. Surgical indication was individualized and based on the patient's age and neurological status, hematoma size and location, and related mass effect (Fig. 4). The remaining 81 patients $(11 \%)$ underwent clinical, neurological, and neuroradiological monitoring and received only medical therapy. Thirty-nine of these patients underwent

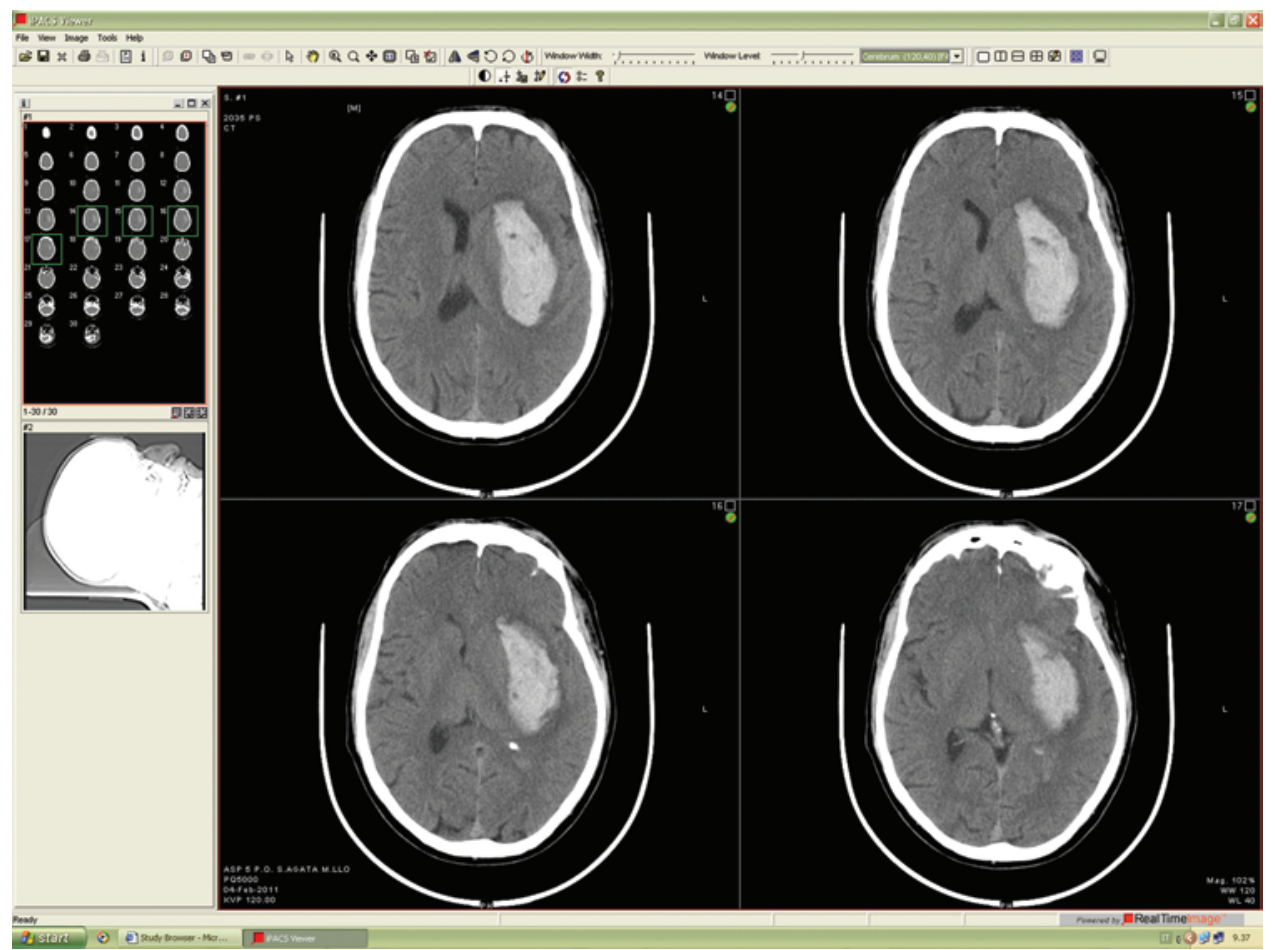

FIG. 2. Telemedicine screenshot showing multiple slices of brain CTs obtained in a 45-year-old patient with spontaneous nucleocapsular ICH. Telemedicine allowed rapid visualization of the CT and the collection of relevant clinical data. In general, nucleocapsular hematoma was not managed by central transfer, unless there was evidence of relevant mass effect with possible deterioration requiring neurointensive care or surgical control of ICP. Cautious conservative management led to a good outcome in this case. 
Telemedicine for intracerebral hemorrhage

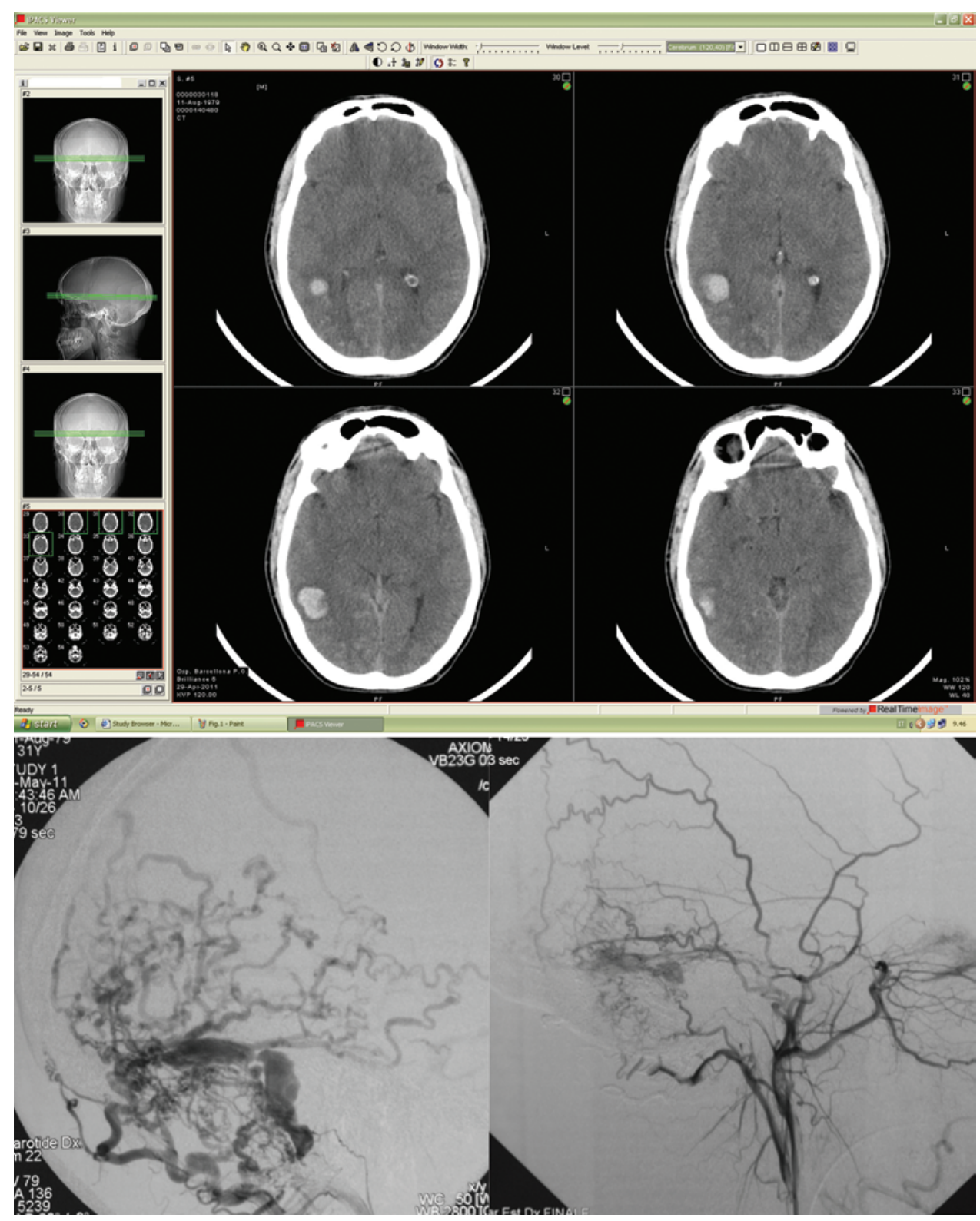

FIG. 3. Upper: Brain CT scans obtained in a 27-year-old patient with spontaneous lobar ICH. Telemedicine screenshot reproduces the CT console of the peripheral hospital with visualization of the scouts, multiple slices of the examination, and tools for image management. In general, hematoma without evidence of mass effect was managed at the peripheral hospital, unless one suspected vascular malformations, tumors, or sinus thrombosis, requiring urgent management. In such cases, clinical data collection is fundamental to avoid disastrous delay of diagnosis and treatment. Lower: Angiograms showing evidence of a dural arteriovenous fistula (left), which was promptly managed with embolization (right).

further studies for suspected vascular malformations, tumors, or sinus thrombosis. Suspicion was confirmed in 21 patients. In terms of efficacy of interpretation, clinical and radiological data were misinterpreted in 26 cases, including 8 cases of secondary centralization and 18 cases of inappropriate centralization, corresponding to $3.5 \%$ of the whole series.

\section{Discussion}

Results suggested that the care of patients with $\mathrm{ICH}$ through a telemedicine system is safe and effective. The telemedicine network allowed prompt selection of a subgroup of patients who were the best candidates for direct neurosurgical or neurointensive care. This group consisted of $24 \%$ of all patients with ICH who had been admitted to the peripheral hospitals and included those (13\%) in whom the surgical control of ICP was readily necessary or decidedly predictable and those $(11 \%)$ in whom bleeding due to vascular malformations, tumors, or sinus thrombosis had to be ruled out in the acute setting. The interpretation of images and clinical data was correct in $96.5 \%$ of cases.

The selection of patients as candidates for direct 


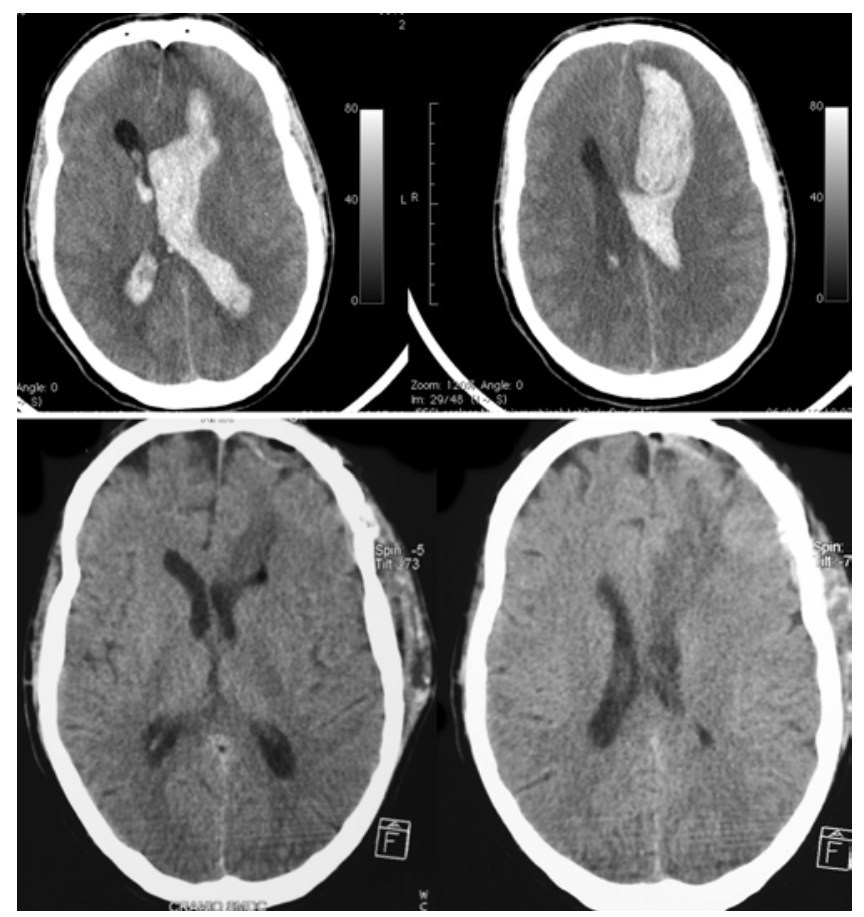

FIG. 4. Upper: Computed tomography scans obtained with the telemedicine system, showing ICH and IVH in a 24-year-old patient. Cases of large lobar hemorrhage in young patients were centralized to undergo emergency CTA and surgical treatment. Lower: Computed tomography scans obtained after emergency surgical treatment.

neurosurgical care is controversial, and the practice in ICH care continues to be arbitrary. , $^{8,11,16,21}$ This attitude is in part a consequence of the misinterpretation of the results of the largest trial on spontaneous ICH management, the International Surgical Trial in Intracerebral Haemorrhage (STICH). ${ }^{15}$ Evidence of the poor efficacy of surgical management for spontaneous ICH persuaded many surgeons that there is no need to perform surgery in patients with ICH. However, it is evident that early relief of elevated ICP is effective in all other clinical settings. Furthermore, a detailed post hoc analysis of the data in the STICH showed that $42 \%$ of patients had an associated IVH, and the prognosis in most of these cases is dismal. Removing these patients from the analysis and focusing on superficial ICHs, the data presented a more encouraging picture for surgery, with $49 \%$ of patients gaining a favorable outcome versus $37 \%$ of the conservatively treated patients $(\mathrm{p}=0.080){ }^{16}$

Beyond the data mentioned above, there are specific categories of patients with spontaneous ICH who remain reasonably suitable candidates for surgery, such as those with acute hydrocephalus due to intraventricular bleeding and those with a large $(>3 \mathrm{~cm})$ cerebellar hemorrhage who are neurologically deteriorating or have brainstem compression and/or hydrocephalus. ${ }^{5}$ Furthermore, seemingly spontaneous ICHs may hide vascular abnormalities (aneurysm, arteriovenous malformation, dural arteriovenous fistula, and cavernous malformation), tumors, or venous thrombosis, which require specific surgical treatments, or vasculitis and coagulopathy, which require specific medical treatments. A qualified judgment of the combined clinical status of the patients and the neuroradiological picture is therefore essential to avoid inappropriate management.

Telemedicine networks represent a new approach to improve stroke care in community settings. Since the first 1999 description by Levine and Gorman ${ }^{14}$ of a telemedicine network for the treatment of patients with ischemic stroke (Telestroke), several studies have been conducted to demonstrate the efficacy of telemedicine in providing specialist care to patients with ischemic stroke in rural communities. Telemedicine clearly showed its efficacy in providing hospitals in rural communities with the expertise of established stroke centers on demand and within minutes. ${ }^{14,17,19}$ Moreover, it has been demonstrated that, embedded in a comprehensive stroke network, the teletransfer of clinical information and brain images yields medical results similar to those following treatment at experienced stroke centers. . $^{2,23}$

In particular, it has been shown that Telestroke improves the care of patients with ischemic stroke by limiting the number of interhospital referrals, ${ }^{3}$ allowing one to choose or exclude candidates for thrombolytic therapy with intravenous tissue plasminogen activator ${ }^{7}$ and eventually improve neurological outcome in these patients. ${ }^{3,18}$ Actually, using the Telestroke approach, intravenous thrombolysis with tissue plasminogen activator has been shown to be safe and has been applied more frequently than without teleneurological consultation. , $2,4,24^{\text {Nonethe- }}$ less, studies focused on the role of telemedicine in the treatment of patients with ICH in acute settings are lacking. Only 1 study demonstrated that telemedicine might enhance enrollment of patients with ICH into time-sensitive stroke trials, such as FAST (Factor Seven for Acute Hemorrhagic Stroke), a multicenter randomized trial to study the role of recombinant factor VIIa in $\mathrm{ICH}{ }^{26}$

Our telemedicine network (RESPECT) considerably improved the rapidity of a second opinion consultation, making it more efficient in acute settings. The possibility of directly viewing neuroradiological images and doing postprocessing analysis made neurosurgical consultation easier and faster, allowing us to choose patients who needed transfer to the academic hub and making their transfer more rapid. Indeed, our data confirm that telemedicine allows one to effectively organize selected and timely interhospital transfers to specialized institutions. ${ }^{1}$

As a second step, we evaluated the role of telemedicine in optimizing the care of patients with $\mathrm{ICH}$ at peripheral hospitals in the acute setting. Five hundred forty-nine patients $(74.9 \%)$ could be directly treated at the spoke hospitals. They underwent clinical, neurological, and neuroradiological monitoring based on neurosurgical indications; they also underwent more CT studies than the patients admitted to the hub, despite similar clinical conditions. We did not address the safety and efficacy of the procedure by measuring the long-term outcome. Actually, in general it is possible that patients treated at the peripheral hospitals received different levels of care. Nevertheless, our experience suggests that in the acute and subacute settings, telemedicine allows the treatment of patients with ICH directly at the peripheral hospitals with the opportunity for online neurosurgical evaluation. This 
may contribute to increase competence in ICH management among health system professionals and optimization of resources, avoiding unnecessary transfers. Patients treated at the spokes were those who needed standard care or those in whom supportive care was indicated. Nonetheless, 8 patients $(1.4 \%)$ treated at the spoke hospitals presented with a rapid and unpredicted worsening of neurological status and/or a neuroradiological picture that made secondary transfer to the referring neurosurgical department necessary. In 5 of 8 patients, the worsening was a result of treatment with oral anticoagulants. As demonstrated already, the use of such drugs can increase the risk of spontaneous ICH or hemorrhagic evolution in patients with ICH. There are lessons to be learned from these cases. To avoid secondary transfer, history and clinical/neurological examination by emergency physicians at the peripheral hospitals should be as accurate as possible to make neurosurgical consultation easier and more precise. Moreover, one must strictly follow neurosurgical indications, respecting time intervals between radiological examinations and repeatedly using the telemedicine system for a second opinion. Even if those needing secondary transfer were a small proportion, the patients in a deep coma or moribund status were not centralized and were only given supportive care. Thus, the rate of secondary transfer may underestimate the patient deterioration that occurred at the spoke hospitals.

\section{Conclusions}

Telemedicine may represent a new approach for the treatment of patients with ICH in the acute and subacute settings. Allowing rapid visualization and interpretation of neuroradiological data, telemedicine brought to rural community hospitals the expertise of established stroke centers on demand and within minutes. When necessary, patient referrals via telemedicine were faster. In the remaining cases, the treatment of patients with $\mathrm{ICH}$ at the peripheral hospitals was possible, avoiding unnecessary and costly transfers. This in some way contributed to increased competence among health system professionals, showing a significant educational impact. Nevertheless, the interpretation of data was inaccurate in $3.5 \%$ of the cases, with a small percentage of patients treated at the peripheral hospitals who had a secondary deterioration in their neurological status or neuroradiological picture and a group of patients who were inappropriately transferred to the hub. Secondary referrals can be avoided by implementing the transfer of clinical data to make neurosurgical consultation easier and more precise.

\section{Disclosure}

The authors report no conflict of interest concerning the materials or methods used in this study or the findings specified in this paper.

Author contributions to the study and manuscript preparation include the following. Conception and design: Angileri. Acquisition of data: Raffa. Drafting the article: Conti, Cardali. Approved the final version of the manuscript on behalf of all authors: Conti. Study supervision: Tomasello.

\section{References}

1. Audebert HJ, Kukla C, Clarmann von Claranau S, Kühn J, Vatankhah B, Schenkel J, et al: Telemedicine for safe and extended use of thrombolysis in stroke: the Telemedic Pilot Project for Integrative Stroke Care (TEMPiS) in Bavaria. Stroke 36:287-291, 2005

2. Audebert HJ, Kukla C, Vatankhah B, Gotzler B, Schenkel J, Hofer S, et al: Comparison of tissue plasminogen activator administration management between Telestroke Network hospitals and academic stroke centers: the Telemedical Pilot Project for Integrative Stroke Care in Bavaria/Germany. Stroke 37:1822-1827, 2006

3. Audebert HJ, Moulin T: Telestroke: the use of telemedicine in stroke care. Preface. Cerebrovasc Dis 27 (Suppl 4):V-VI, 2009

4. Audebert HJ, Schwamm L: Telestroke: scientific results. Cerebrovasc Dis 27 (Suppl 4):15-20, 2009

5. Broderick J, Connolly S, Feldmann E, Hanley D, Kase C, Krieger D, et al: Guidelines for the management of spontaneous intracerebral hemorrhage in adults: 2007 update: a guideline from the American Heart Association/American Stroke Association Stroke Council, High Blood Pressure Research Council, and the Quality of Care and Outcomes in Research Interdisciplinary Working Group. Stroke 38:2001-2023, 2007

6. Broderick JP, Brott TG, Duldner JE, Tomsick T, Huster G: Volume of intracerebral hemorrhage. A powerful and easy-touse predictor of 30-day mortality. Stroke 24:987-993, 1993

7. Demaerschalk BM, Hwang HM, Leung G: Cost analysis review of stroke centers, telestroke, and rt-PA. Am J Manag Care 16:537-544, 2010

8. Dubourg J, Messerer M: State of the art in managing nontraumatic intracerebral hemorrhage. Neurosurg Focus 30(6):E22, 2011

9. Fogelholm R, Murros K, Rissanen A, Avikainen S: Long term survival after primary intracerebral haemorrhage: a retrospective population based study. J Neurol Neurosurg Psychiatry 76:1534-1538, 2005

10. Gebel JM, Broderick JP: Intracerebral hemorrhage. Neurol Clin 18:419-438, 2000

11. Gregson BA, Mendelow AD: International variations in surgical practice for spontaneous intracerebral hemorrhage. Stroke 34:2593-2597, 2003

12. Hess DC, Wang S, Gross H, Nichols FT, Hall CE, Adams RJ: Telestroke: extending stroke expertise into underserved areas. Lancet Neurol 5:275-278, 2006

13. Hess DC, Wang S, Hamilton W, Lee S, Pardue C, Waller JL, et al: REACH: clinical feasibility of a rural telestroke network. Stroke 36:2018-2020, 2005

14. Levine SR, Gorman M: "Telestroke:" the application of telemedicine for stroke. Stroke 30:464-469, 1999

15. Mendelow AD, Gregson BA, Fernandes HM, Murray GD, Teasdale GM, Hope DT, et al: Early surgery versus initial conservative treatment in patients with spontaneous supratentorial intracerebral haematomas in the International Surgical Trial in Intracerebral Haemorrhage (STICH): a randomised trial. Lancet 365:387-397, 2005

16. Mendelow AD, Gregson BA, Mitchell PM, Murray GD, Rowan EN, Gholkar AR: Surgical trial in lobar intracerebral haemorrhage (STICH II) protocol. Trials 12:124, 2011

17. Meyer BC, Raman R, Chacon MR, Jensen M, Werner JD: Reliability of site-independent telemedicine when assessed by telemedicine-naive stroke practitioners. J Stroke Cerebrovasc Dis 17:181-186, 2008

18. Meyer BC, Raman R, Ernstrom K, Tafreshi GM, Huisa B, Stemer AB, et al: Assessment of long-term outcomes for the STRokE DOC Telemedicine Trial. J Stroke Cerebrovasc Dis [epub ahead of print], 2010

19. Meyer BC, Raman R, Hemmen T, Obler R, Zivin JA, Rao R, et 
al: Efficacy of site-independent telemedicine in the STRokE DOC trial: a randomised, blinded, prospective study. Lancet Neurol 7:787-795, 2008

20. Minutoli F, Angileri FF, Conti A, Herberg A, Aricò D, Baldari $\mathrm{S}$, et al: Timing of examination affects reliability of $99 \mathrm{mTc}-$ methoxyisobutylisonitrile SPECT in distinguishing neoplastic from nonneoplastic brain hematomas. J Nucl Med 46: 574-579, 2005

21. Pouratian N, Kassell NF, Dumont AS: Update on management of intracerebral hemorrhage. Neurosurg Focus 15(4):E2, 2003

22. Sairanen T, Soinila S, Nikkanen M, Rantanen K, Mustanoja S, Färkkilä M, et al: Two years of Finnish Telestroke: thrombolysis at spokes equal to that at the hub. Neurology 76:11451152,2011

23. Schwab S, Vatankhah B, Kukla C, Hauchwitz M, Bogdahn U, Fürst A, et al: Long-term outcome after thrombolysis in telemedical stroke care. Neurology 69:898-903, 2007

24. Schwamm LH, Rosenthal ES, Hirshberg A, Schaefer PW, Little EA, Kvedar JC, et al: Virtual TeleStroke support for the emergency department evaluation of acute stroke. Acad Emerg Med 11:1193-1197, 2004
25. Strehle EM, Shabde N: One hundred years of telemedicine: does this new technology have a place in paediatrics? Arch Dis Child 91:956-959, 2006

26. Switzer JA, Hall CE, Close B, Nichols FT, Gross H, Bruno A, et al: A telestroke network enhances recruitment into acute stroke clinical trials. Stroke 41:566-569, 2010

27. Wang S, Lee SB, Pardue C, Ramsingh D, Waller J, Gross H, et al: Remote evaluation of acute ischemic stroke: reliability of National Institutes of Health Stroke Scale via telestroke. Stroke 34:e188-e191, 2003

Manuscript submitted December 14, 2011.

Accepted January 27, 2012.

Please include this information when citing this paper: DOI: 10.3171/2012.1.FOCUS11356.

Address correspondence to: Alfredo Conti, M.D., Ph.D., Department of Neurosurgery, University of Messina, Via Consolare Valeria, 1, 98125 Messina, Italy. email: alfredo.conti@unime.it. 